\title{
Detection of bipolar disorder ${ }^{\dagger}$
}

\author{
Allan $\mathrm{H}$. Young and Holly MacPherson
}

\begin{abstract}
Summary
Major depressive episodes are common in bipolar disorder, which consequently may be misdiagnosed as major depressive disorder. Improved detection of bipolar disorder rests upon better ascertainment of a history of hypomania. Antidepressants are of dubious benefit in bipolar disorder
\end{abstract}

and more accurate diagnosis of depression would promote better treatment.

\section{Declaration of interest}

None.
Allan Young (pictured) is Chair of Psychiatry at Imperial College London where he is also Director of the Centre for Mental Health. Holly MacPherson is a research assistant at the Institute of Mental Health, University of British Columbia, vancouver, Canada.

\section{General review of field}

Mood disorders are responsible for $12 \%$ of the total burden of all disease and are associated with enormous personal, societal and economic costs. ${ }^{1}$ This group of disorders includes both major depressive and bipolar disorder, with major depressive episodes being prominent in both. Major depressive disorder is the commonest mood disorder, with lifetime prevalence rates reported up to $16 \% .^{2}$ Bipolar disorder includes a number of subtypes including bipolar I disorder, bipolar II disorder and bipolar spectrum. The differentiation between these subtypes essentially rests on the severity of the pathological elevations of mood, with mania being unique to bipolar I disorder and hypomania characteristic of bipolar II disorder and bipolar spectrum, although full major depressive episodes do not occur in bipolar spectrum. The prevalence rate of bipolar disorders is generally thought to be considerably lower than that of major depressive disorder. Typically, population surveys using structured diagnostic interviews and standardised criteria report lifetime prevalence rates to be $1.0 \%$ for bipolar I disorder, $1.1 \%$ for bipolar II disorder, and $2.4 \%$ for subthreshold bipolar disorders. ${ }^{3}$ However, rates of bipolar disorder reported in extensive community surveys and those from prospective longitudinal clinical studies have been inconsistent. This is an indication that accurate lifetime prevalence estimates may be underestimated in community surveys. The inclusion criteria and the diagnostic thresholds for bipolar disorder have been suggested by prospective cohort studies to be too restrictive to adequately recognise bipolar spectrum in the general population, notably in young adults when the disorder is in its early stages. The exact epidemiology of bipolar spectrum disorder therefore remains somewhat uncertain, although recent studies clearly indicate it may affect up to $5 \%$ of the general population at some point in their lives. ${ }^{3}$

\section{Major depressive episodes: bipolar or major depressive disorder?}

Major depressive episodes are central clinical states for both major depressive disorder and bipolar disorder. The distinction between 'bipolar' and 'unipolar' types of depressive episodes has been

'See pp. 49-56, this issue. disputed since at least the late 1800s. Instead of differentiating between episodic categories, Kraepelin, the father of current psychiatric nosology, used the term manic-depressive illness to describe people with recurrent mood episodes, even if these episodes were all depressive. ${ }^{4}$ The distinction of major depressive and bipolar disorders as separate illnesses evolved from the broad classification of manic-depressive illness in the middle of the last century. ${ }^{5}$ This distinction has become established in standard international diagnostic systems, such as the ICD and the DSM, since the 1980s. According to one current classification system, DSM-IV-TR, major depressive disorder is defined by at least one experience of a major depressive episode characterised by symptoms of low mood, along with low self-esteem, and/or loss of interest or pleasure in normally enjoyable activities. ${ }^{6}$ Bipolar disorder is characterised by at least one episode of mania or hypomania and individuals usually also suffer from recurring major depressive episodes. ${ }^{6}$ During depressive episodes, the clinical presentation of bipolar disorder may include psychosis, diurnal mood variation and hypersomnia more frequently than in major depressive disorder. In addition, people with bipolar disorder may experience more depressive episodes of short duration than individuals with major depressive disorder. Clinicians should recognise these features as potentially indicative of bipolar disorder; ${ }^{7}$ however, these characteristics are not diagnostic. The key feature, therefore, which distinguishes bipolar disorder from major depressive disorder, is the presence or history of mania or hypomania.

Prevailing operational diagnostic systems of psychiatric illnesses are categorical and were developed to enhance the reliability of diagnoses in both clinical and research settings. These classifications have major limitations and some individuals cannot be neatly classified under a particular category and are classified under the vague rag-bag of 'not otherwise specified'. A dimensional approach provides an alternative model to the categorical approach. The dimensional method places people along a gradient instead of defining them as belonging to discrete entities with clear boundaries. A dimensional model may also incorporate pertinent details such as impairment, severity, chronicity and outcome. Although the dimensional approach has been recently critiqued, ${ }^{8}$ the upcoming DSM-5 is likely to be a hybrid of categorical classifications coupled with dimensions; whether this will represent an overall advance on current systems remains to be seen, although the potential utility for mood disorders is clear.

There have been a number of recent studies on people with apparent major depressive disorder who after more detailed enquiry clearly have some bipolar symptoms. As a result of the difficulty of obtaining a history of hypomania, bipolar II disorder and bipolar spectrum are frequently underrecognised. Depressive 
symptoms occur much more commonly than do manic or hypomanic symptoms and these latter symptoms tend to be underreported. In contrast to when in a depressive state, those experiencing hypomania rarely complain of, or directly suffer from, their changes in energy, activity and sleep behaviour; instead, these changes are often viewed as positive experiences.' Quite often the presentation at the initial onset of bipolar disorder is a depressive episode and many people with bipolar disorder have multiple episodes of major depression prior to their first episode of mania; ${ }^{10}$ this clearly indicates a need for a high index of suspicion for bipolar disorder in adolescent, as well as adult, depression. Persistent hypomanic and manic symptoms have been found to be a risk state in adolescence for clinically relevant bipolar disorder, ${ }^{11}$ although recognition of a bipolar diagnosis may be delayed by up to 10 years.

\section{Smith et al's study}

Most of the studies that have reported the misdiagnosis of bipolar disorder as major depressive disorder have been conducted in secondary or tertiary care settings whereas Smith et al, in this issue of the Journal, attempted to corroborate these results in a primary care setting in the UK. ${ }^{12}$ In Smith et als sample of people with a working diagnosis of 'unipolar' depression, up to $21.6 \%$ of these individuals could be diagnosed with unrecognised bipolar disorder using the Hypomania Checklist (HCL-32) $)^{13}$ and the Bipolar Spectrum Diagnostic Scale (BSDS). ${ }^{14}$ Despite having low positive predictive values for detecting DSM-IV bipolar disorder, the authors suggest using screening instruments such as the HCL-32 and BSDS for detecting broadly defined hypomania. Similar to previous findings, it was found that subthreshold features of bipolar disorder were relatively common in individuals with supposed unipolar depression (in primary, secondary and tertiary care settings) and were associated with a more severe pattern of depressive illness and greater psychosocial disability and quality of life impairments.

Although laudable as the first study in a UK primary care setting to identify the misdiagnosis of bipolar disorder in people diagnosed with unipolar depression, Smith et als study has several limitations. One major weakness was the low return rate of HCL-32 and BSDS questionnaires; of the 3117 individuals invited to participate, only $18.5 \%$ volunteered and returned their questionnaires. Those who did volunteer in the study were found to be significantly more likely to be taking antidepressant medications as well as being older than those who did not volunteer. There is a possibility that because of the age difference, the volunteer groups contained more participants with bipolar disorder, thus inflating the findings. In addition, the volunteer group could have demonstrated more severe depressive illnesses or may have been more likely to report hypomanic symptoms. On the other hand, the authors used the DSM-IV as the gold standard for diagnosis. The DSM-IV embodies restrictive diagnostic criteria and may not aid the identification of subtle hypomanic symptoms, and thus leads to reduced rates of diagnosis of bipolar disorder.

These findings may have major implications for treatment strategies for people with major depressive disorder with unrecognised hypomanic symptoms. A misdiagnosis of bipolar disorder as major depressive disorder may lead to unsuitable antidepressant monotherapy. As a result, individuals who have received an inaccurate diagnosis may have poorer outcomes and a worse course of illness that may include a higher proclivity for chronic and recurrent mood episodes, increased rates of subsyndromal symptoms and greater impairment of psychosocial functioning. Furthermore, there is a notable risk of antidepressant monotherapy triggering treatment-emergent mood switches. In those with an inaccurate diagnosis, especially those with bipolar II disorder, this may be made worse by the long-term use of antidepressant medications. Recently, this risk has caused clinicians to be discouraged by treatment guidelines from the use of antidepressants for bipolar depression, although such treatment remains common throughout the world. Currently, antidepressants are the most commonly prescribed monotherapy for people with bipolar disorder in the USA with approximately $50 \%$ of individuals being prescribed initial and long-term treatment in this manner, ${ }^{15}$ and this pattern is likely to be common worldwide.

The key message is clear: improved detection of bipolar disorder is important and relatively simple with screening tools such as the HCL-32. This applies to primary care as well as to specialist services and, if undertaken, is likely to form the basis of more appropriate treatment regimens and thus improved outcomes for individuals.

Allan H. Young, FRCPsych, Centre for Mental Health, Imperial College London; Holly MacPherson, BSC, Institute of Mental Health, University of British Columbia, vancouver, Canada

Correspondence: Allan H. Young, Centre for Mental Health, Imperial College London, St Dunstan's Road, London W6 8RP, UK. Email: a.young@Imperial.ac.uk

First received 21 Dec 2010, final revision 14 Mar 2011, accepted 6 Apr 2011

\section{References}

1 Andrews G, Titov N. Depression is very disabling. Lancet 2007; 370: 808-9

2 Kessler RC, Berglund P, Demler O, Jin R, Koretz D, Merikangas KR, et al. The epidemiology of major depressive disorder: results from the national comorbidity survey replication (NCS-R). JAMA 2003; 289: 2095-3105.

3 Merikangas KR, Akiskal HS, Angst J, Greenberg PE, Hirschfeld RMA, Petukhova $\mathrm{M}$, et al. Lifetime and 12-month prevalence of bipolar spectrum disorder in the National Comorbidity Survey Replication. Arch Gen Psychiatry 2007; 64: 543-52.

4 Trede K, Salvatore P, Baethge C, Gerhard A, Maggini C, Baldessarini RJ. Manic-depressive illness: evolution in Kraepelin's Textbook, 1883-1926. Harv Rev Psychiatry 2005; 13: 155-78.

5 Woodruff RA Jr, Guze SB, Clayton PJ. Unipolar and bipolar primary affective disorder. Br J Psychiatry 1971; 119: 33-8.

6 American Psychiatric Association. Diagnostic and Statistical Manual of Mental Disorders (4th edn, text revision) (DSM-IV-TR). APA, 2000.

7 Forty L, Smith D, Jones L, Jones I, Caesar S, Cooper C, et al. Clinical differences between bipolar and unipolar depression. Br J Psychiatry 2008; 192: $388-9$.

8 Lawrie SM, Hall J, McIntosh AM, Owens DGC, Johnstone EC. The 'continuum of psychosis': scientifically unproven and clinically impractical. Br J Psychiatry 2010; 197: 423-5.

9 Angst J, Gamma A, Benazzi F, Ajdacic V, Eich D, Rössler W. Toward a redefinition of subthreshold bipolarity: epidemiology and proposed criteria for bipolar-II, minor bipolar disorders and hypomania. J Affect Disord 2003; 73: $133-46$.

10 Duffy A, Alda M, Hajek T, Grof P. Early course of bipolar disorder in high-risk offspring: prospective study. Br J Psychiatry 2009; 195: 457-8.

11 Tijssen MJA, van Os J, Wittchen H-U, Lieb R, Beesdo K, Mengelers R, et al. Prediction of transition from common adolescent bipolar experiences to bipolar disorder: 10-year study. Br J Psychiatry 2010; 196: 102-8.

12 Smith DJ, Griffiths E, Kelly M, Hood K, Craddock N, Simpson SA. Unrecognized bipolar disorder in primary care patients with depression. Br J Psychiatry 2011; 199: 49-56.

13 Angst J, Adolfsson R, Benazzi F, Gamma A, Hantouche E, Meyer TD, et al. The $\mathrm{HCL}-32$ : towards a self-assessment tool for hypomanic symptoms in outpatients. J Affect Disord 2005; 88: 217-33.

14 Ghaemi SN, Miller CJ, Berv DA, Klugman J, Rosenquist KJ, Pies RW. Sensitivity and specificity of a new bipolar spectrum diagnostic scale. J Affect Disord 2005; 84: 273-7.

15 Baldessarini RJ, Leahy L, Arcona S, Gause D, Zhang W, Hennen J. Patterns of psychotropic drug prescription for US patients with diagnoses of bipolar disorders. Psychiatr Serv 2007; 58: 85-91. 